\title{
MONTE CARLO SIMULATIONS OF THE ENERGY RESOLUTION FUNCTION OF n_TOF AT CERN
}

C. Carrapiço ${ }^{1,2}$, E. Berthoumieux ${ }^{2}$, I. F. Gonçalves ${ }^{1}$, F. Gunsing ${ }^{2}$, A. Mengoni ${ }^{3}$, P. Vaz ${ }^{1}$, V. Vlachoudis ${ }^{3}$ and the n_TOF Collaboration ${ }^{4}$

${ }^{1}$ Instituto Tecnológico e Nuclear: Estrada Nacional 10, 2686-953 Sacavém - Portugal

${ }^{2}$ CEA / Saclay, DSM / IRFU / SPHN, F-91191 Gif-sur-Yvette, France

${ }^{3}$ European Organization for Nuclear Research: CERN CH-1211 Genève 23 Switzerland

${ }^{4}$ www.cern.ch/ntof

The n_TOF facility ${ }^{1}$ is a time-of-flight spectrometer dedicated to study neutron induced reactions, mainly neutron capture and fission cross sections. The spectrometer consists of a pulsed proton beam $\left(7 \times 10^{12}\right.$ protons per pulse, $6 \mathrm{~ns}$ width, $\left.20 \mathrm{GeV} / \mathrm{c}\right)$ impinging on a $80 \times 80 \times 60 \mathrm{~cm}^{3}$ lead target. The neutrons produced by spallation reactions reach the detector station at $185 \mathrm{~m}$ through an evacuated tube. There, neutron induced reactions are studied by using the time-of-flight technique. The facility is unique for its high instantaneous neutron flux (of the order $10^{6}$ neutrons $/ \mathrm{cm}^{2} /$ proton pulse at 185 meters), an excellent energy resolution, low background conditions, and a very low duty cycle. This combination allows one to measure neutron capture and fission cross-sections in the energy range from $1 \mathrm{eV}$ to $250 \mathrm{MeV}$ with high precision.

For the analysis of the data in the resolved resonance region up $1 \mathrm{MeV}$, a precise and accurate knowledge of the distribution of the energy resolution is mandatory. The only way to obtain the resolution function in a detailed way is to use Monte Carlo simulations together with the experimental verification with well-known resonance reactions at selected energies. Such calculations and an analytical fit of the results have been performed for the target setup of the first phase of data taking.

In this study, Monte Carlo simulations performed for the assessment and comparison of the resolution function for different target configurations are reported. The different resolution functions are compared and discussed. 


\section{INTRODUCTION / MOTIVATION}

The n_TOF target (Ref. 1) was designed with a $80 \times 80 \times 60 \mathrm{~cm}^{3}$ spallation core made of lead surrounded by water that serves the purpose of cooling and moderation. Two studies have been done

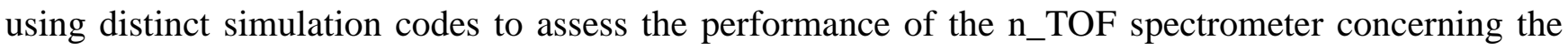
resolution function. In one of the studies, described in Ref. 2, the resolution function was simulated for energies below $20 \mathrm{MeV}$ using the code CAMOT and implementing the geometry described in Ref. 3. In the other study described in Ref. 4 , the code FLUKA ${ }^{5}$ was used with the same geometry. Both studies consider a moderation depth of $5 \mathrm{~cm}$. The resolution function is calculated at the sample position which is located at about $185 \mathrm{~m}$ from the moderation target. Because of the large geometrical factor involved in the problem the resulting statistics were limited. Recently, during a check of the spallation target, it was found that during the construction a supplementary O-ring was introduced just behind the aluminum windows that seal the target system from the time-of-flight spectrometer increasing the moderation depth to a value of about $5.8 \mathrm{~cm}$ at the center of the target. The knowledge of the effects of this extra moderation depth and its influence on the energy resolution function as well as the neutron energy spectrum is crucial for the data analysis. The accurate knowledge of the energy resolution function is essential for the analysis in the resolved resonance region using an R-matrix code consisting of fitting the resonance shape. In order to study the effect of the increased moderation depth of $5.8 \mathrm{~cm}$ with high statistics we performed new simulations with the code MCNPX. To study the consistency of our results, we also performed the simulation with a moderation depth of $5 \mathrm{~cm}$ and compared the results with the resolution available from the two previous simulations.

\section{RESOLUTION FUNCTION}

Neutrons are generated by spallation reactions in the target and are afterwards moderated until they 
reach the evacuated neutron tube. The unknown path followed by the neutrons inside the target and associated moderation system, impacts the assessment of the neutron energy using the time-of-flight technique by introducing an intrinsic uncertainty. The goal for determining the neutron energy resolution function is to describe and include the energy-dependent resolution. The analytical description of the resolution function used is this work is already implemented in the R-matrix analysis code SAMMY (Ref. 6) with the RPI broadening function. Many of the parameters of this analytical function are energy-dependent. The simulated resolution function can be adapted to this function by adjusting the energy dependence of the parameters. The effect of the resolution is most visible in the reaction cross sections in the resolved resonance region by a tail which visibly deforms the overall shape of the resonance and shifts the apparent peak energy. The RPI function we have used has the following form (Eq. 1):

Where the parameters $\Lambda, \tau, \mathrm{A}_{1}, \mathrm{~A}_{3}$ and $\mathrm{A}_{5}$ are functions of the neutron energy. The parameter $\mathrm{A}_{1}$ equals zero if the sum of the two exponentials is negative.

In Eq. 1 the $\chi^{2}$ distribution can be related to the moderated neutrons and the exponential tails are associated with the combined target-moderator system.

\section{SIMULATION}

Monte Carlo simulations with the code MCNPX have been performed in order to assess the differences introduced by the increased moderation thickness. A detailed geometrical description of the neutron production system considering all the components inside the target container was used (Fig. 1). The neutron beam pipe used for the time-of-flight spectrometer was also implemented. In addition, two ideal tube collimators and two ideal beam colimators were included in order to fully describe the experimental conditions encountered in the experimental area. The first tube collimator is located at $\sim 71 \mathrm{~m}$ from the exit plan of the target system and the second at $\sim 140 \mathrm{~m}$ and they serve the purpose of 
killing particles traveling outside the beam tube. The beam collimators are located at $\sim 137 \mathrm{~m}$ and $\sim 175$

m. The detailed description of the beam pipe and the position of the collimator can be found in Ref. 1.

III.A. State of the Art

The neutrons were generated by spallation reactions triggered by $20 \mathrm{GeV}$ protons impinging on a lead block (the target). The preequilibrium and evaporation physics associated with the spallation processes was simulated using the CEM2k (Ref. 7) model implemented in MCNPX 2.6e (Ref. 8). The CEM2k model is able to describe fission reactions and the production of light fragments heavier than ${ }^{4} \mathrm{He}$, using the Generalized Evaporation Model code (Ref. 9, 10). It contains also a number of improvements and refinements in the cascade and Fermi break-up models comparing with previous models used in MCNPX simulation.

All these features permit us to describe quite well a large variety of spallation, fission and fragmentation reactions necessary for the simulations performed.

In addition to this we used one DXTRAN sphere (Ref. 8, 11). This variance reduction technique available in MCNPX is capable of dealing with the large geometrical factor related to the transport of particles over large distances without interactions in simple geometries like the TOF tube. This technique is based on the angle-biasing technique together with deterministic transport of the neutrons inside the pipe (Ref. 11).

III.B. Simplifications in the Simulation

The interaction of a $20 \mathrm{GeV}$ proton with a lead block produces a huge amount of particles including neutrons. The neutron production per proton is approximately 300 neutrons. This large neutron production ratio combined with the cascade of nuclear reactions produced inside the target and the 
associated particles produced yields a heavy and lengthy simulation problem. The problem is characterized by a very high multiplicity of particles and a reduced solid angle. However, the neutrons that are of relevance for our studies are those which are produced in the forward direction, in a very narrow solid angle. In order to increase the simulation speed without compromising the realistic approach of the simulation concerning the experimental conditions, several simplifications have been

made. Considering that we are interested only in low-energy neutrons ( $1^{-9}$ to $\left.10 \mathrm{MeV}\right)$, only hadronic particles were tracked in the simulation. To compensate for the very small solid angle $(<1 \mathrm{mrad})$ due to the $185 \mathrm{~m}$ length of the neutron beam pipe and collimators, we have used a DXTRAN sphere to achieve good statistics.

III.C. Simulated Results

The neutron energy and equivalent distance (path length equivalent to the time spent by a neutron of a given outgoing velocity inside the target-moderator system) were tallied using a user defined tally used in similar calculations (Ref. 12) for the facility GELINA. These quantities are stored in a 2D matrix (Fig. 2) to facilitate the analysis.

The tally binning consists of one bin per millimeter for the equivalent distance and of ten bins per decade of energy. The equivalent distance was tracked from -10 to $300 \mathrm{~cm}$ and the energy range considered is between $1 \mathrm{eV}$ to $10 \mathrm{MeV}$.

\section{ANALYSIS}

IV.A. Procedure and Methodology

The simulated data for $5 \mathrm{~cm}$ and $5.8 \mathrm{~cm}$ of moderation depth where fitted using the RPI resolution 
function and the results compared with two previous simulations data assessed for $5 \mathrm{~cm}$ using the FLUKA and CAMOT Monte Carlo simulation codes. The fit was done using two different energy bins ( 2 and 10 bins per decade) for the data of FLUKA and MCNPX. Although it was necessary to rebin the FLUKA data in the equivalent distance axis to achieve reasonable statistics, the results showed that the fit with 10 bins per decade agrees very well with the fit using 2 bins per decade. The same was observed for the data from MCNPX. For the data obtained with CAMOT, the fit was processed using 2 bin per decade in the energy scale due to statistical constraints. We have compared the Full Width at Half Maximum (FWHM) and the Full Width at a Tenth of the Maximum (FWTM) of the fitted energy resolution function for each energy interval.

In order to determine the parameters of the resolution function, we have fitted the equivalent distance for each energy bin using the RPI function with all parameters free in order to obtain the best description of the simulated data. The fitted RPI function for each energy bin is analyzed and the quantities FWHM and FWTM are extracted. Figure 3 displays the RPI fitted function for the $80 \mathrm{eV}$ bin overlayed on the $5 \mathrm{~cm}$ data simulated with MCNPX. The resolution function is fitted throughout the whole energy range and analyzed.

IV.B Analysis of the FWHM and FWTM

The procedure used to assess the differences between the resolution functions assessed with different codes and for the different depths of moderation was the comparison of the FWHM and the FWTM for each energy bin between the different sets of data. The plotted data of the FWHM and FWTM variation with the energy is provided in Fig. 4, 5 and 7 respectively.

From Fig. 4 and Fig.5 it is possible to see that the results from the previous simulations are different at higher neutron energies. Also the results of this work are not consistent with any of the two previous simulations. 
Concerning the simulated equivalent distance using MCNPX, and moderation depths of 5 and $5.8 \mathrm{~cm}$ with water, the behaviors in almost identical for the equivalent distance spectrum. This can be seen in Fig. 6, where the simulated data is displayed both 0n a linear and logarithmic scale.

The FWHM and FWTM (Fig. 7) assessed show very small differences over the energy range for the two different moderation depths.

\section{CONCLUSION}

The results obtained in this study show the existence of important differences between the different simulations done to assess the resolution of the $\mathrm{n}_{-} \mathrm{TOF}$ spectrometer. The reason behind these differences is not clear and may be related to the different codes, libraries and methodologies used. The transportation of the particles through the TOF tube can be a cause, as all codes/methodologies were different. With MCNPX, the transport of the particles in the TOF tube was done during the simulation using the code's variance reduction techniques. For the FLUKA simulation, the neutron spectra was tallied just outside the target system and then transported with a separated code trough the TOF tube. Results obtained in this study using MCNPX show that an increase in moderation depth introduces almost no changes in the equivalent distance shape. This leads to the importance of validating the simulations and determine which one reflects the characteristics of the n_TOF spectrometer. As one can see from Fig. 9, the energy resolution function dominates the broadening of the resonance shape above several $\mathrm{keV}$. A change in the resolution function used in the analysis of resolved resonances will certainly affect the extracted resonance parameters. On the other hand, since the area of the resonance is conserved, an integrated quantity like the Maxwellian averaged capture cross section will not change with a different resolution function. Work is currently being done to verify the adequacy of the simulations by analyzing well known resolved resonances from data taken at the n_TOF facility. 


\section{ACKNOWLEDGMENTS}

This work was partially funded by the Portuguese Foundation for Science and Technology (FCT), under projects PDCT/FP/63929/2005 and POCI/FP/81931/2007. The financial support provided to the construction of the TOF spectrometer by the European Commission's $5^{\text {th }}$ Framework Program under contract FIKW-CT-2000-00107 is acknowledged.

\section{REFERENCES}

1. C. Borcea et. al., "The neutron time of flight facility at CERN" Journal of Nuclear Science and Technology, Supplement 2653 (2002)

2. C. Coceva et. al., "On the figure of merit in neutron time-of-flight measurements" Nuclear Instruments and Methods in Physics Research A Vol. 489, p. 346-356 (2002)

3. S. Andriamonje et al., Neutron TOF Facility (PS 213), Technical Design Report, CERN Report CERN/INTC/2000-004, 2000.

4. V. Vlachoudis, et. al., "Monte Carlo simulation of the neutron time-of-flight facility at CERN", Advanced Monte Carlo for radiation physics, particle transport simulation and applications, Lisbon, Portugal, 23-26 Oct 2000, p. 1175-1180

5. FLUKA: a multi-particle transport code, A. Fassò, A. Ferrari, J. Ranft, and P.R. Sala, CERN2005-10 (2005), INFN/TC_05/11, SLAC-R-773

6. SAMMY - Multilevel R-Matrix Fits to Neutron and Charged-Particle Cross-Section Data Using Bayes' Equations, Oak Ridge National Laboratory

7. S. G. Mashnik et. al., "Improved Intranuclear Cascade Models for the Codes CEM2k and LAQGSM" International Conference on Nuclear Data for Science and Technology, Santa Fe, New Mexico, 26 September - 1 October 2004, Vol. 769, p. 1188-1192, American Institute of Physics (2005) 
8. Monte Carlo N-Particle Transport Code version 2.6e, Los Alamos National Laboratory

9. Furihata, S., Ph.D. thesis, Tohoku University, Japan (2003); Nucl. Instr. Meth., B171, 252 (2000).

10. Furihata, S.,"The GEM Code - the Generalized Evaporation Model and the Fission Model", Advanced Monte Carlo for Radiation Physics, Particle Transport Simulation and Applications. Proceedings of the Monte Carlo 2000 Conference held in Lisbon, 23-26 October, 2000. Berlin Heidelberg: Springer-Verlag, 2001., p.1045

11. A Sample Problem for Variance Reduction in MCNP, Report LA-10363-MS, Los Alamos National Laboratory12. M. Flaska, et. al., "Modeling of the GELINA neutron target using coupled electron-photon-neutron transport with the MCNP4C3 code", Nuclear Instruments and Methods in Physics Research Section A, Vol. 531, p. 392-406

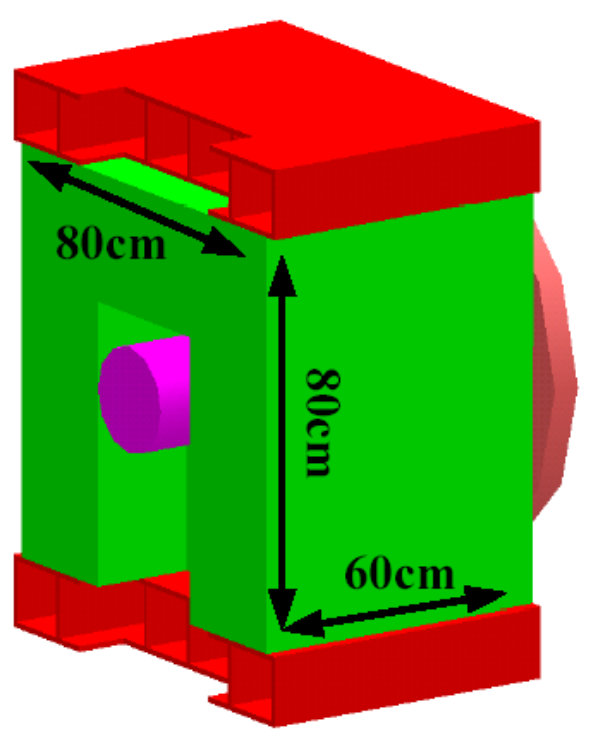

Fig. 1. Target system accompanied by the supporting structure and aluminum entrance window in the right. The proton beam impinges from the left side. 


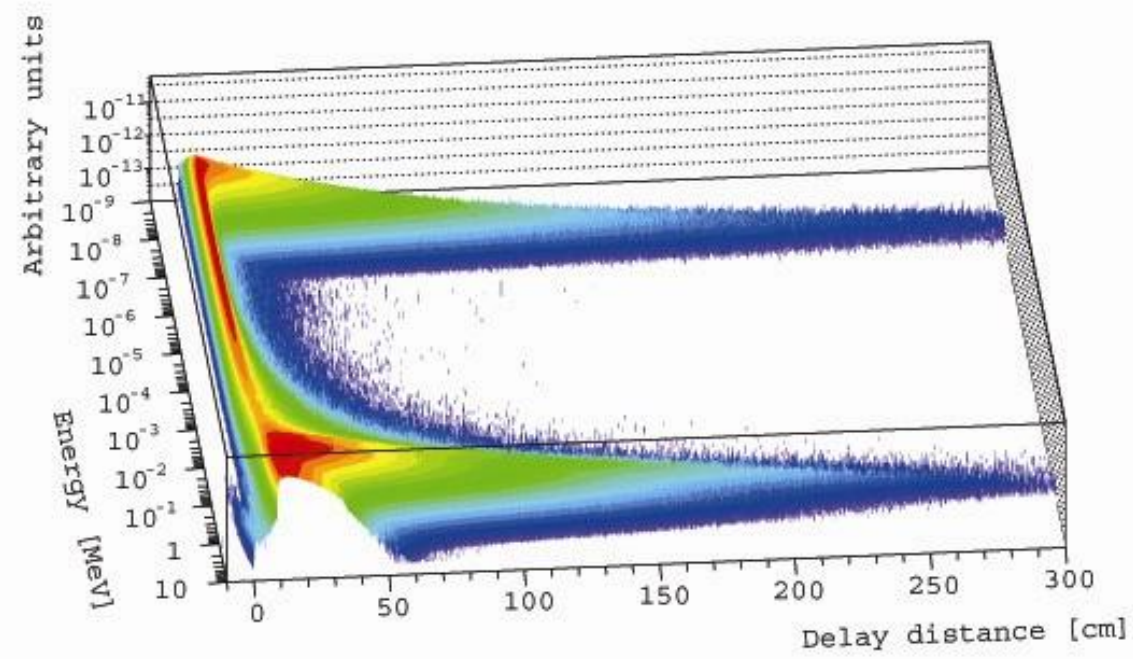

Fig. 2. 2D matrix of the tallied quantities in MCNPX, showing their dependence.
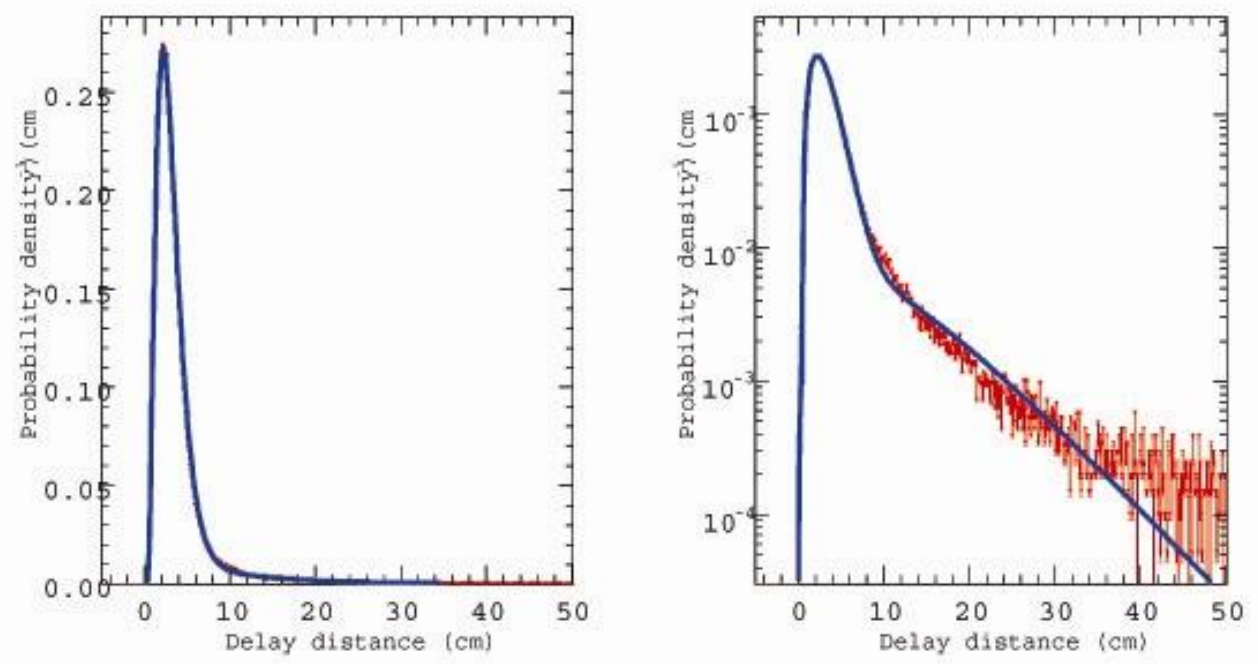

Fig. 3. Fit of the simulated data simulated with MCNPX at $80 \mathrm{eV}$ for a moderation depth of $5 \mathrm{~cm}$. 

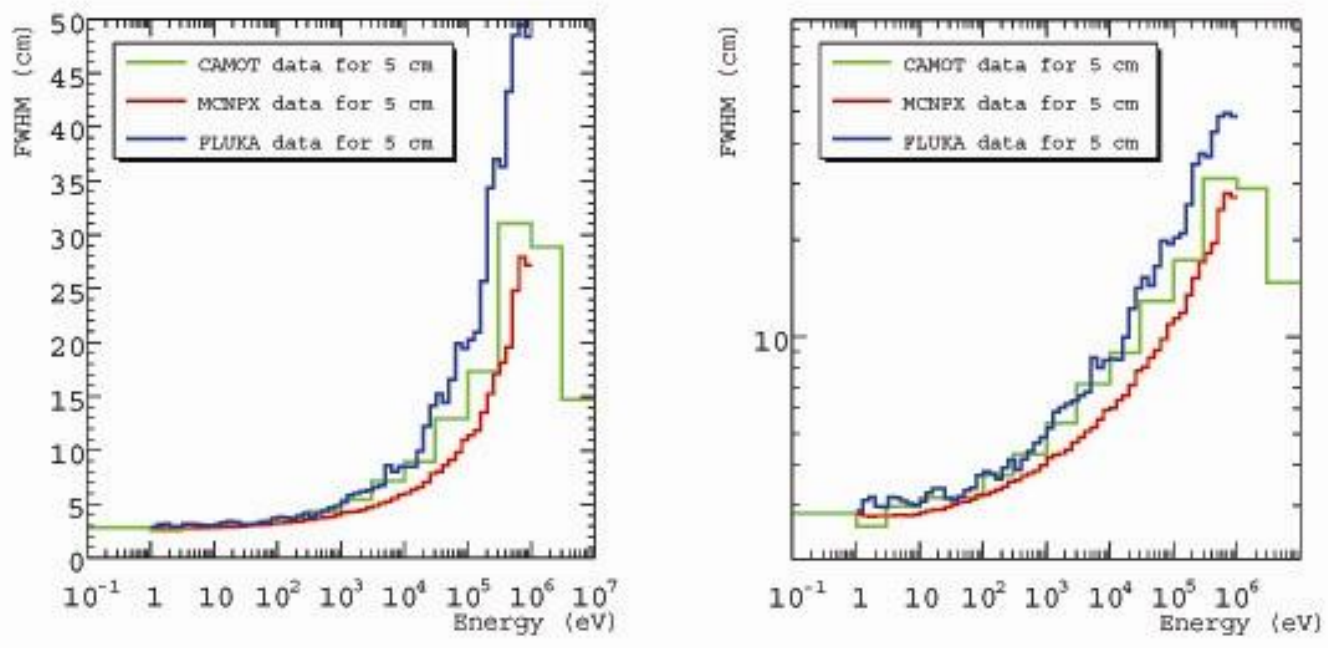

Fig. 4. FWHM for the three parameterizations for $5 \mathrm{~cm}$ of water.
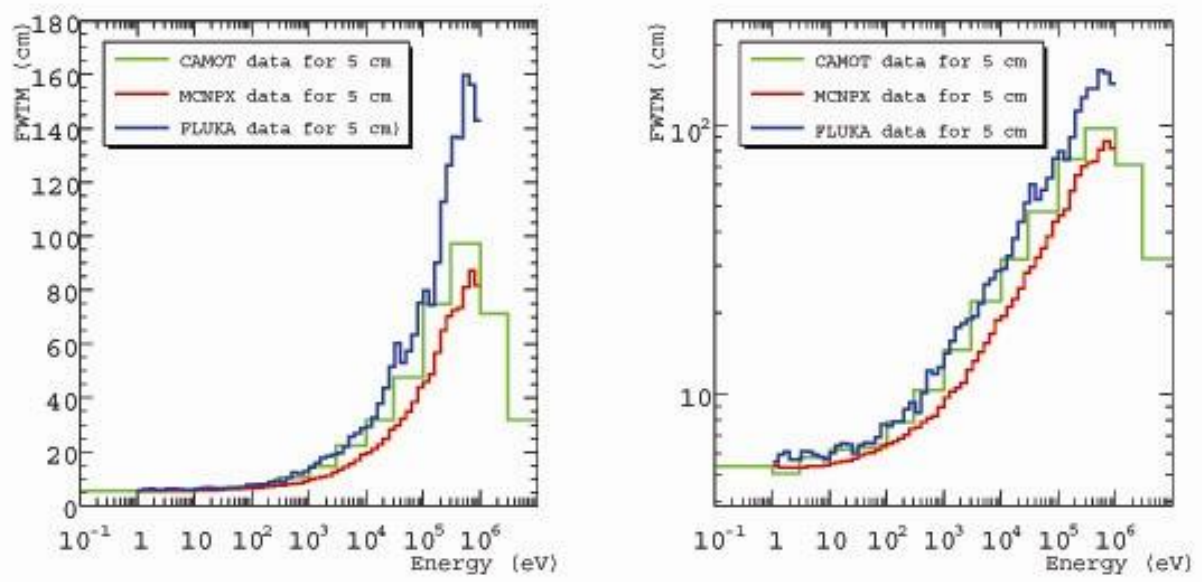

Fig. 5. FWTM for the three parameterizations for $5 \mathrm{~cm}$ of water. 

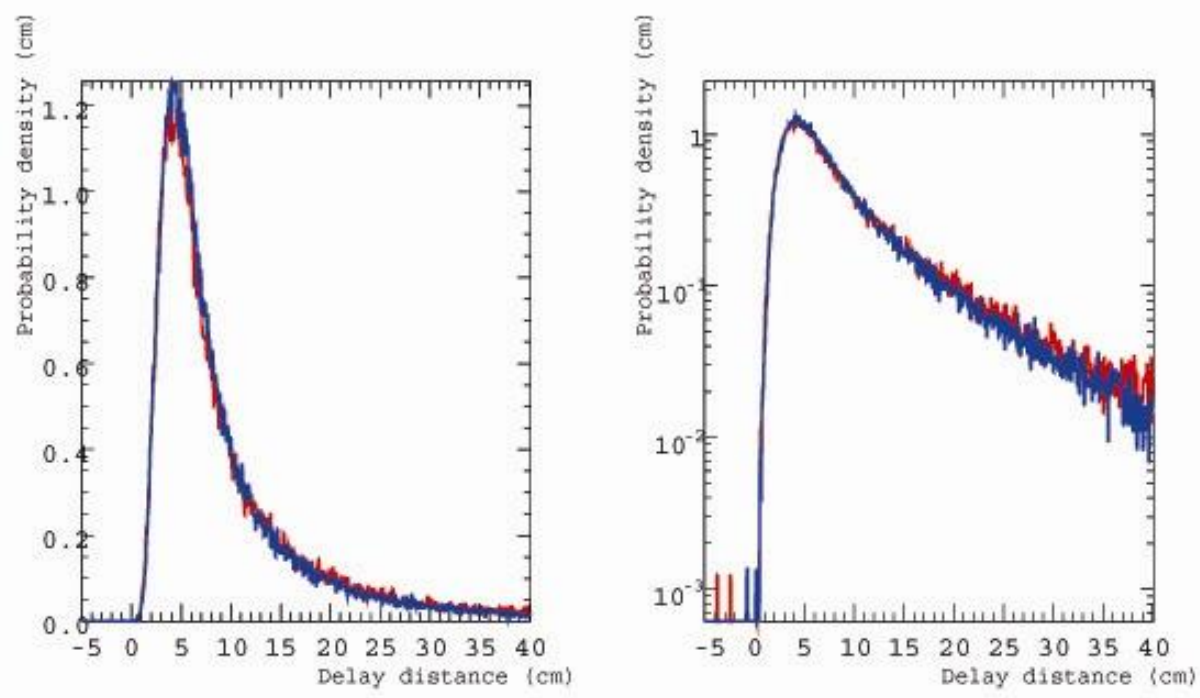

Fig. 6. Comparison of the equivalent distance between two different moderation depths for $8 \mathrm{keV}$ (red for $5 \mathrm{~cm}$ and blue for $5.8 \mathrm{~cm}$ ).
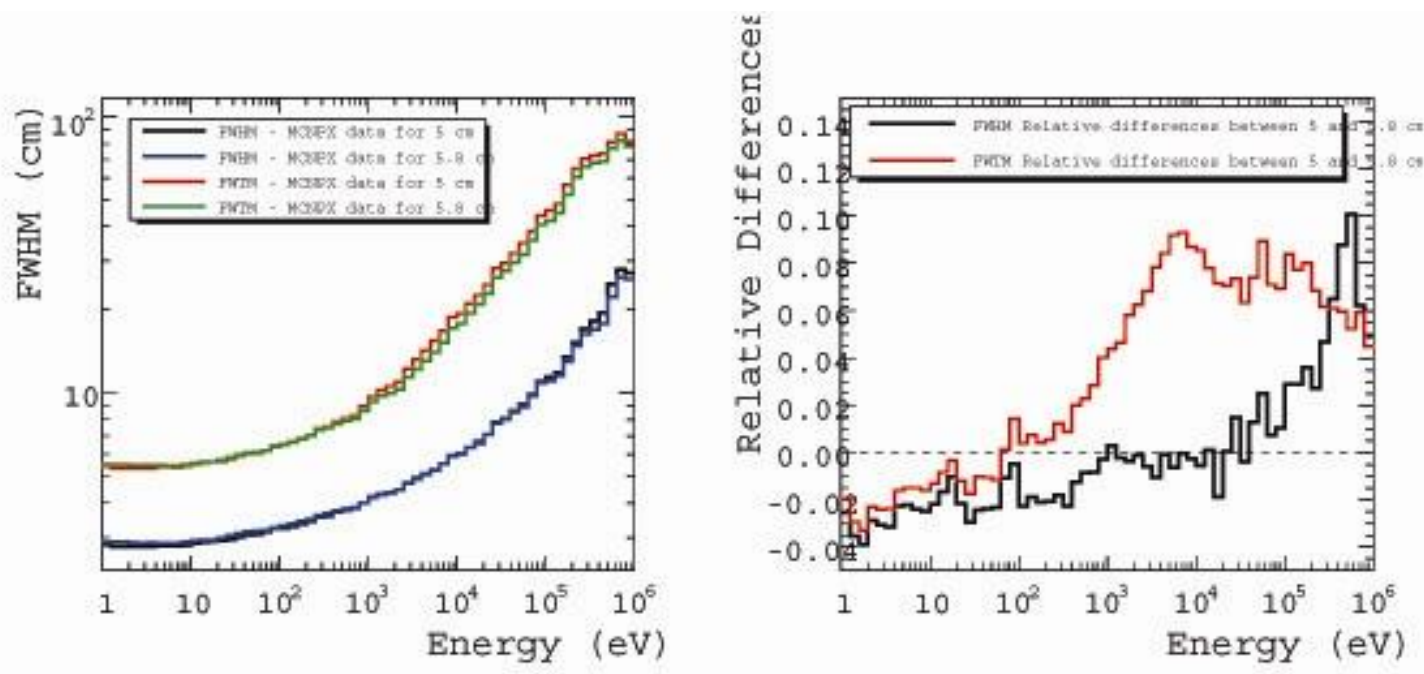

Fig. 7. FWHM and FWTM for 5 and $5.8 \mathrm{~cm}$ of moderation depth simulated with MCNPX. [plot ratio?] 


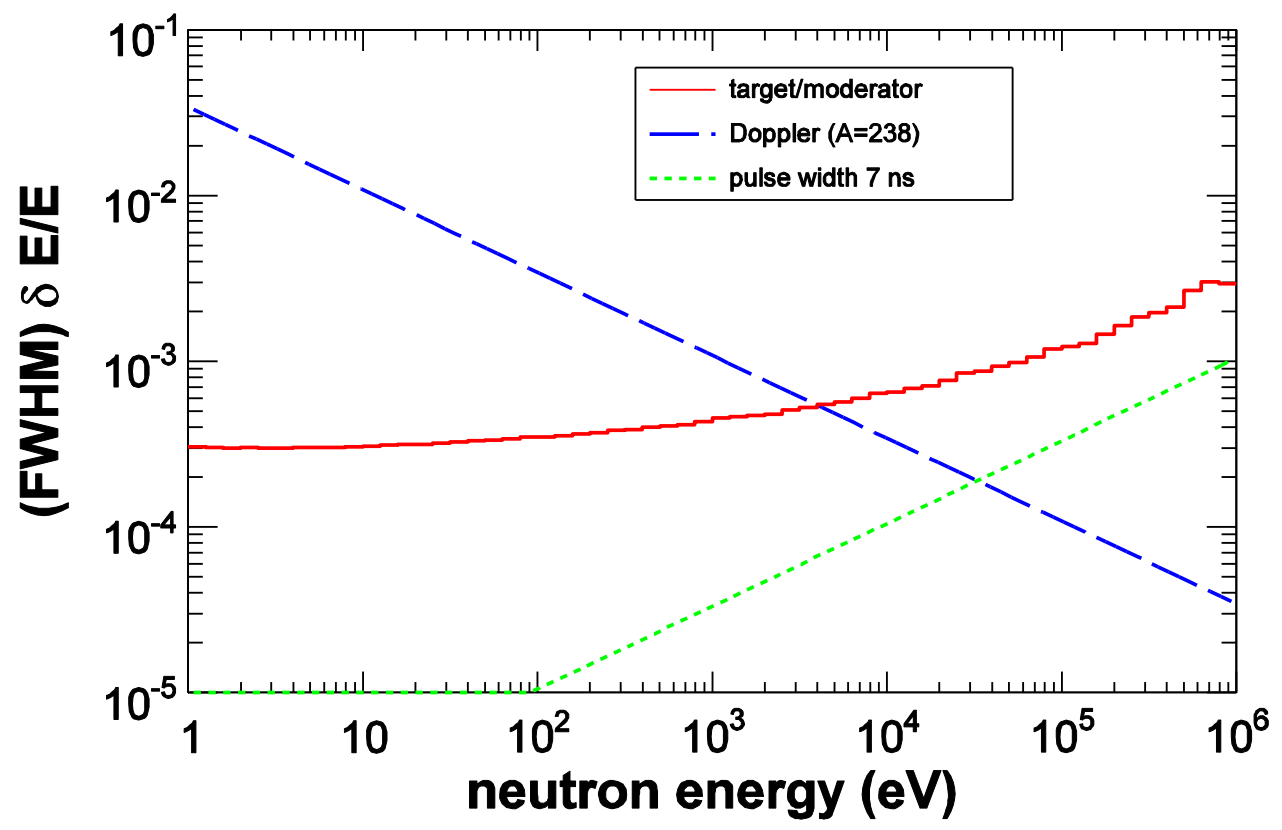

Fig. 8. Different contributions for the broadening of the resonances. The target/moderator curve represents the results obtained for the FWHM with MCNPX using a moderation depth of $5 \mathrm{~cm}$. 